\section{Epidermolysis bullosa acquisita: current diagnosis and therapy}

\author{
Christine R. Mehren, ${ }^{1}$ Robert Gniadecki \\ 'Department of Dermatology, Bispebjerg \\ Hospital, Copenhagen; \\ 2University of Copenhagen, Bispebjerg \\ Hospital, Denmark
}

\begin{abstract}
Epidermolysis bullosa acquisita (EBA) is an acquired, autoimmune subepidermal blistering disease with an approximate prevalence of $0,2 /$ million people. The hallmark of EBA is the presence of autoantibodies (mainly IgG class) to anchoring fibril collagen (type VII collagen) located at the dermal-epidermal junction. Clinically EBA is subdivided into the inflammatory and the non-inflammatory phenotypes, depending on the level of the cleavage in the basal membrane. A recent addition to the diagnostic techniques is the analysis of the serration pattern of the autoantibody deposits at the basal membrane in the direct immunofluorescence. EBA and the closely related bullous systemic lupus erythematosus are the only diseases presenting with the so-called u-serration pattern which distinguishes them from many other autoimmune subepidermal blistering diseases. We also discuss the recent advances in therapy, including the experience with Rituximab.
\end{abstract}

\section{Clinical features}

Epidermolysis bullosa acquisita (EBA) was described for a century ago by Ellliot. ${ }^{1}$ It is a rare disease with an approximate prevalence of $0.2 /$ million people. ${ }^{2}$ EBA is an acquired, autoimmune cutaneous subepidermal blistering disease that primarily involves the skin, and sometimes mucous membranes. There is no racial or gender predilection. EBA often presents in the fourth to fifth decades of life. The hallmark of EBA is the presence of autoantibodies (mainly IgG class) to type VII collagen, a major component of anchoring fibrils at the dermal-epidermal junction. The disease occurs in approximately 5\% of unselected patients with basement membrane zone antibodies. ${ }^{3}$ The blister-inducing potential of autoantibodies to type VII collagen have been shown by demonstrating their capacity to trigger an Fc $\gamma$ dependent inflammation leading to split formation in cryosections of human skin. ${ }^{4}$ Clinically EBA is subdivided into two clinical types: the inflammatory and the non-inflammatory phenotype. ${ }^{5}$ Patients with the noninflammatory form of EBA (the classical EBA type) have increased skin fragility with subse- quent formation of blisters or erosions on the trauma-prone areas of the skin, such as extensor surfaces of elbows, knees, ankles, and buttocks. Tense vesicles and bullae appear on noninflamed skin or scarred skin. Nail dystrophy and scarring alopecia have been observed in some patients with the classical EBA. The inflammatory form of EBA can mimic almost all other chronic bullous diseases, and its clinical differentiation from bullous pemphigoid, mucous membrane pemphigoid and linear IgA bullous dermatosis may be difficult. ${ }^{6}$ It presents with widespread, tense vesicles and bullae and is not localized to trauma-prone sites and generally heals with minimal scarring and milia formation. Progressive and recurrent disease in the mucosal tissues can result in irreversible complications similar to those seen in mucous membrane pemphigoid (MMP) including blindness and oesophageal strictures. ${ }^{7}$ By electron microscopy, the cleavage plane can be seen within the lamina lucida or sub-lamina densa regions of the dermal-epidermal junction. If the cleavage is within the lamina lucida, it is associated with the presence of an inflammatory infiltrate rich in polymorphonuclear neutrophils. ${ }^{8}$ The deeper level of split in the non-inflammatory EBA type may explain why it usually heals with significant scar and milia formation, which is only rarely observed in the inflammatory type (Table 1).

\section{Diagnostic techniques}

The first diagnostic criteria for EBA were established in the early 1970 s by Roenigk and associates. They were i) spontaneous or trauma-induced blisters resembling hereditary dystrophic EB, ii) adult onset, iii) a negative family history for EB, and iv) the exclusion of all other bullous diseases. ${ }^{17}$ However, since then at least 33 verified cases of childhood EBA have been reported in the literature, mainly of the inflammatory subtype. ${ }^{18}$ In addition a diagnosis of EBA cannot be done reliably solely by clinical findings, because of the variable clinical and histological presentations.

Immunofluorescence techniques remains the cornerstone of the diagnosis of EBA and have increasingly replaced the immunoelectron microscopy (IEM) as the gold standard..$^{19}$ Other investigative diagnostic techniques are immunoblotting, ELISA, and immunoprecipitation. Direct Immunofluorescence (DIF) on perilesional skin demonstrates linear immune deposits of immunoreactants, mainly IgG, at the basement membrane zone. However deposits of IgA, IgM, C3, C4, or properdin may be detected as well. In EBA depositions of IgG in the absence of $\mathrm{C} 3$ is seen more commonly than in BP. Furthermore, deposits of multiple conjugates (including IgG, IgA, IgM, C3, C4, or properdin) are seen more frequently in the setting of EBA. ${ }^{7}$ Indirect immunofluorescence (IIF) can detect the presence of circulating IgG
Correspondence: Christine R. Mehren, Department of Dermatology, Bispebjerg Hospital, Copenhagen, Denmark.

E-mail: c_mehren@hotmail.com

Key words: bullous pemphigoid, epidermolysis bullosa, serration pattern, immunofluorescence.

Received for publication: 14 April 2011.

Accepted for publication: 9 August 2011.

This work is licensed under a Creative Commons Attribution NonCommercial 3.0 License (CC BYNC 3.0).

(C) Copyright C.R. Mehren and R. Gniadecki., 2011 Licensee PAGEPress, Italy

Dermatology Reports 2011; 3:e38

doi:10.4081/dr.2011.e38

autoantibodies, directed against type VII collagen in the basement membrane, it usually detects the IgG autoantibodies that binds to the dermal floor on salt split skin. Salt split skin substrate can be used to distinguish EBA and bullous pemphigoid (BP), because IgG autoantibodies from patients with bullous pemphigoid bind to the epidermal roof (upper part) of salt-split skin. If the antibody labels the dermal side of the separation, the patient usually has either EBA or bullous SLE (there are, however, other diseases with dermal staining; anti-epiligrin (lamina-5), Cicatricial pemphigoid, Chan's disease, Zilliken's disease and Ghohestani's disease). ${ }^{19}$

A breakthrough in the diagnostics was the observation of specific serration patterns in the autoantibody deposits at the basement membrane in direct immunofluorescence. Besides a true linear staining pattern, two DIF immunodeposition patterns have been described i) a u-serrated staining pattern is typical of EBA, and ii) an n-serrated staining pattern in other subepidermal immunobullous diseases. ${ }^{9}$ The binding of autoantibodies in EBA to type VII collagen, can ultrastructurally be seen as upstanding arms between the rootlets of the basal keratinocytes, resulting in u shapes. Consequently these two distinct patterns can be applied in order to differentiate EBA from other pemphigoid variants by DIF only. The type of serration in the case of IgA and IgG is similar, however, in the case of IgA it is more easily recognized due to better fluorescence image contrast. Diseases such as IgA-mediated EBA and inflammatory EBA may look like BP, however, using the serration patterns algorithm, patients with EBA who otherwise would have been erroneously diagnosed can be detected.

Immunoelectron microscopy (IEM) documents the localization of the immune deposits within the dermal-epidermal junction of the skin of EBA patients. By direct immunoelec- 
Table 1. Comparison of the clinical and immunopathological features of subepidermal blistering diseases.

\begin{tabular}{|c|c|c|c|c|}
\hline $\begin{array}{l}\text { Subepidermal immunobullous } \\
\text { disease }\end{array}$ & $\begin{array}{l}\text { Clinical Characteristic } \\
\text { features }\end{array}$ & DIF & $\begin{array}{l}\text { Binding on } \\
\text { salt split skin }\end{array}$ & $\begin{array}{l}\text { Serration } \\
\text { pattern }\end{array}$ \\
\hline $\begin{array}{l}\text { Bullous } \\
\text { pemphigoid (BP) }\end{array}$ & $\begin{array}{l}\text { Elderly, most common autoimmune blistering } \\
\text { disease. Tense blisters on inflammed or non } \\
\text { inflamed skin. Pruritus common, variable } \\
\text { severity. More common in patients with multiple }\end{array}$ & $\begin{array}{l}\text { IgG and } \mathrm{C} 3 \text { or } \mathrm{C} 3 \text { alone }( \pm \\
\text { weaker staining IgM, IgA) at } \\
\text { the dermal-epidermal junction. }\end{array}$ & $\begin{array}{l}\text { Epidermal } \\
\text { (few dermal) }\end{array}$ & n-serrated ${ }^{9}$ \\
\hline
\end{tabular}
sclerosis.

Predilection sites: the inner or anterior thighs, groins, flexor surfaces of the upper extremities and lower abdomen. Oral mucosal lesions are rare.

Pemphigoid

gestationis

Lichen planus

Lichen planus

pemphigoides

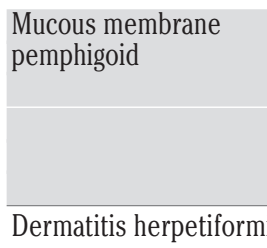

Linear IgA disease

Linear IgA/gG bullous dermatosis ${ }^{12}$ Resemble the lesions of BP, annular vesicobullous lesions with frequent involvement of the oral mucosa.

Predilection sites: no specific

\begin{tabular}{ll} 
Anti-p450-pemphigoid $^{13}$ & Only one case in literature, Fujiwara et al $l^{14}$ \\
\hline Anti-p200 pemphigoid $^{32}$ & $\begin{array}{l}\text { Often resembles BP, could resemble dermatitis } \\
\text { herpetiformis, linear IgA disease or EBA. Could } \\
\text { involve mucous membranes. Often coexisting } \\
\text { psoriasis. } \\
\text { (rapid response to treatment) }\end{array}$
\end{tabular}

Epidermolysis bullosa acquisita Classical EBA: skin fragility, trauma- induced blisters and erosions.

Predilection sites: extensor surfaces of the extremities.

\pm mucous membrane lesion.

Inflammatory EBA: widespread eruptions of tense

blisters on erythematous or normal appearing skin.

Predilection sites: usually on flexural and/or intertriginous areas. \pm mucous membrane lesions

Bullous SLE

Mainly adult patients. Tense blisters on normal or erythematous skin, eruptions usually in a herpetiform arrangement in patients with SLE. Pruritus may be severe.

Predilection sites: trunk and flexural surfaces. Frequently oral lesions.

Anti-p105-pemphigoid ${ }^{16}$
Bullae and erosions on mucous membrane and skin, resembling toxic epidermal necrolysis or pemphigus vulgaris ${ }^{13}$
Linear or granular depositions of $\operatorname{IgG}( \pm \operatorname{IgM}, \operatorname{IgA}, \mathrm{C} 3)$ at the dermal-epidermal junction. antilamin 332 MMP10: linear anti-BP 180 MMP IgG, \pm C3 occasionally IgA.

In ocular MMP:11 linear IgA $\pm \operatorname{IgG}$.

Granular papillary and basement membrane IgA.

Linear deposition of $\mathrm{C} 3$

Epidermal

As BP; epidermal junction.

n-serrated

Linear deposits of IgG at the dermal-epidermal

Epidermal

Unknown anti-BP 180 MMP
and ocular MMP

n-serrated ${ }^{9}$

Dermal in antilamin 332 MMP

Negative

Irrelevant
Linear IgA (rarerly granular) at

Epidermal

n-serrated ${ }^{9}$

Only one case in literature,

Linear IgG and C3 at the dermal- Dermal

Epidermal (few Probably dermal or both n-serrated epidermal and dermal)

\section{Linear IgG ( \pm IgA, IgM), C3 junction.}

Dermal (rarely epidermal or

Dermal u-serrated ${ }^{9}$

Unknown

n-serrated ${ }^{9}$ combined binding)
Linear IgG and C3 deposition at the skin basement membrane zone ${ }^{13}$
Dermal $^{13}$

Unknown 
tron microscopy the ultrastructural localization of in vivo-bound $\operatorname{IgG}$ autoantibodies at the basement membrane is documented. With indirect immunoelectron microscopy the binding site of circulating IgG autoantibodies at the basement membrane is detected. IEM detects IgG autoantibodies at the lamina densa and sublamina densa areas of skin basement membrane. Bullous pemphigoid IgG autoantibodies, on the contrary, are localized to the hemidesmosome and upper lamina lucida. ${ }^{20}$

Immunoblotting represents a sensitive detection method for EBA. ${ }^{21}$ EBA sera will bind to a 290-KDa band in Western blots of human skin basement membrane proteins containing type VII collagen, whereas sera from all other primary blistering diseases will not. This band corresponds to a single alpha chain of the type VII collagen homotrimer molecule. ${ }^{22}$ Western blotting differentiates between anti-p200 pemphigoid and EBA. To distinguish between the latter two disorders, patients' sera have to be subjected to Western blotting of extract from human dermis and then react with 200 and 290 $\mathrm{kDa}$ proteins in antip-200 pemphigoid and EBA, respectively. ${ }^{2}$

ELISA (Enzyme-linked immunosorbent assay) documents the specific basement membrane antigen recognized by the patient's IgG circulating autoantibodies. The ELISA method identifies non-denaturated, nonreduced proteins and is more sensitive than immunoblotting, which only detects denatured, reduced proteins. $^{7}$

\section{Treatment}

Compared with other autoimmune blistering diseases, EBA has a decreased responsiveness to therapy. Inflammatory EBA, EBA presenting in children and IgA-EBA, respond more favourably to the conventional treatment approach of high-dose corticosteroids and corticosteroid sparing agents. In most cases of IgA-EBA, the skin lesions respond to therapy with dapsone alone.

Dapsone and low-dose prednisone are usually effective in treating childhood EBA. ${ }^{18}$ However, high doses of corticosteroids are not recommended as maintenance therapies as the adverse effects of corticosteroids are both time and dose dependent.

Non-inflammatory (classical) EBA is often refractory to systemic corticosteroids, azathioprine, methotrexate, and cyclophosphamide. ${ }^{23}$ Therapeutic options that have proven effective in retrospective observations include extracorporeal photochemotherapy (ECP), i.v. immunoglobulin (IVIG), and Rituximab.

The treatment of EBA patients with IVIG has shown encouraging results. In one retrospective analysis two patients with severe EBA were treated with monthly cycles of IVIG. One of the patients had a complete response, defined as absence of lesions for more than 4 weeks without any treatment. The other patient responded to the treatment, however, due to metastatic lung cancer the treatment was discontinued. ${ }^{24}$ Another case describes a patient treated with 6 cycles of IVIG at a dose of $400 \mathrm{mg} / \mathrm{kg}$ per day for 5 consecutive days (repeating the cycle every 4 weeks). After the second cycle, most of the erosions had healed and marked remission was observed during the 6-month follow-up period. The patient did not experience any negative side effects. ${ }^{25}$

Patients that have had unsatisfactory response to steroid, immunosuppressive agents and IVIG may benefit from the therapy with rituximab. This monoclonal antibody reacting against CD20 depletes mature, autoreactive B-cells. Of the reported cases of EBA patients treated with rituximab, either complete remission or very good partial remissions have been reported. In one retrospective analysis the patient, a 58-year old woman, had to be hospitalized due to the severity of EBA. She had extensive cutaneous and oral ulceration, cellulitis and a deep vein thrombosis secondary to immobility.

After four rituximab infusions at a dose of $375 \mathrm{mg} \mathrm{m}^{-2}$, she experienced complete remission. ${ }^{26}$ Another patient, a 54-year-old woman experienced a partial remission within a month after the onset of rituximab. After twelve rituximab infusions at a dose of $375 \mathrm{mg}$ $\mathrm{m}^{-2}$, she experienced almost complete cutaneous clearance with improved oral intake and mobility. ${ }^{27}$ Rituximab can safely be combined with high-dose IVIG, which may exert a synergistic effect and simultaneously protect against serious infection-related adverse events. . $^{28,29}$

Long-term ECP has been reported to induce remission in three patients with drug resistant, aggressive cases of EBA. ${ }^{30} \mathrm{ECP}$ is based on separation of a leukocyte/lymphocyte-enriched cell fraction from the peripheral blood, extracorporeal treatment of the cells with 8MOP/UVA, and subsequent reinfusion of the cells in the patient. The main effects in EBA seem to consist in inhibition of pathogenetic autoantibody production by B lymphocytes and generation of regulatory $\mathrm{T}$ cells.

When treating a patient with EBA, it is important to be aware of coexisting systemic diseases that might influence the choice of therapy.

Among the systemic diseases reported in association with EBA, are malignancies and autoimmune diseases. ${ }^{23}$ Inflammatory bowel disease (IBD) is one of the more common systemic illnesses associated with EBA; $25 \%$ of EBA patients have IBD. ${ }^{31}$

There are a few cases of EBA patients with coexisting psoriasis. ${ }^{32}$ Ultraviolet radiation is not a treatment option in this case as it has been demonstrated that it can induce blistering in patients with EBA. ${ }^{33}$

\section{References}

1. Elliott GT. Two cases of epidermolysis bullosa. J Cutan Genitourin Dis 1895;13:108.

2. Ishii N, Hamada T, Dainichi T, et al. Epidermolysis bullosa acquisita; what's new. J Dermatol 2010;37:220-30.

3. Zhu XJ, Niimi Y, Bystryn JC. Epidermolysis bullosa acquisita. Incidence in patients with basement membrane zone antibodies. Arch Dermatol 1990;126:171-4.

4. Sitaru C, Kromminga A, Hashimoto T, et al. Autoantibodies to type VII collagen mediate Fcgamma-dependent granulocyte activation and induce dermal-epidermal separation in cryosections of human skin. Am J Pathol 2002;161:301-11.

5. Tanka H, Ishida, Yamamoto A, et al. A novel variant of aquired epidermolysis bullosa with autoantibodies against the central triple-helical domain of type VII collagen. Lab Invest 1997;77:623-32.

6. Roengik HH, Ryan JG, Bergfeld WF. Epidermolysis bullosa acquisita. Report of three cases and review of all published cases. Arch Dermatol 1971;103:1-10.

7. Lehman JS, Camilleri MJ, Gibsom LE. Epidermolysis bullosa acquisita: concise review and practical considerations. Int $\mathrm{J}$ Dermatol 2009;48:227-36.

8. Mihai S, Sitaru C. Immunopathology and molecular diagnosis of autoimmune bullous diseases. J Cell Mol Med 2007;11:46281.

9. Vodegel RM, Jonkman MF, Pas HH, De Jong MC. U-serrated immunodeposition pattern differentiates type VII collagentargeting bullous disease from other subepidermal bullous automimmune diseases. Br J Dermatol 2004;151:112-8.

10. Natsuga K, Nishie W, Shinkuma S, et al. Circulating IgA and IgE autoantibodies in antilaminin-332 mucous membrane pemphigoid. Br J Dermatol 2010;162:513-7.

11. Smith EP, Taylor TB, Meyer LJ, Zone JJ. Identification of a basement membrane zone antigen reactive with circulating IgA antibody in ocular cicatricial pemphigoid. J Invest Dermatol 1993;101:619-23.

12. Shimizu S, Natsuga K, Shinkuma $S$, et al. Localized linear IgA/IgG dermatosis. Acta Derm Venereol 2010;90:621-4.

13. Georgi M, Jainta S, Bröcker EB, Zillikens D. Autoantigens of subepidermal bullous autoimmune dermatoses. Hautarzt 2001; 52:1079-89.

14. Fujiwara S, Shinkai H, Takayasu S, et al. A case of subepidermal blister disease associated with autoantibody against $450 \mathrm{kD}$ protein. J Dermatol 1992;19:610-3.

15. Chen KR, Shimizu S, Miyakawa S, et al. Coexistence of psoriasis and an unusual IgG-mediated subepidermal bullous der- 
matosis: identification of a novel 200-kDa lower lamina lucida target antigen. Br J Dermatol 1996;134:340-6.

16. Chan LS, Cooper KD. A novel immunemediated subepidermal bullous dermatosis characterized by IgG autoantibodies to a lower lamina lucida component. Arch Dermatol 1994;130:343-7.

17. Roenigk HH Jr, Ryan JG, Bergfeld WF. Epidermolysis bullosa acquisita. Report of three cases and review of all published cases. Arch Dermatol 1971;103:1-10.

18. Mayuzumi M, Akiyama M, Nishie W, et al. Childhood epidermolysis bullosa acquisita with autoantibodies against the noncollagenous 1 and 2 domains of type VII collagen: case report and review of the literature. Br J Dermatol 2006;155:1048-52.

19. Woodley D, Remington J, Chen M. Autoimmunity to type VII Collagen: Epidermolysis Bullosa Acquisita. Clin Rev Allerg Immunol 2007;33:78-84.

20. Vodegel RM, de Jong MC, Pas HH, Jonkman MF. IgA-mediated epidermolysis bullosa acquisita: Two cases and review of the literature. J Am Acad Dermatol 2002; 6:919-25.

21. Schmidt E, Zillikens D. Research in practice: diagnosis of subepidermal autoim- mune bullous disorders. J Dtsch Dermatol Ges 2009;7:296-300.

22. Woodley DT, Briggaman RA, O'Keefe EJ, et al. Identification of the skin basementmembrane autoantigen in epidermolysis bullosa acquisita. N Engl J Med 1984; 310:1007-13.

23. Hallel-Halevy, Nadelman C, Chen M, Woodley DT. Epidermolysis bullosa acquisita: update and review. Clin Dermatol 2001;19:712-8.

24. Sanli H, Akay BN, Ayyildiz E, et al. Remission of severe autoimmune bullous disorders induced by long-term extracorporeal photochemotherapy. Transf Apheres Sci 2010;43:353-9.

25. Segura S, Iranzo P, Martínez-de Pablo I, et al. High-dose intravenous immunoglobulins for the treatment of autoimmune mucocutaneous blistering diseases: evaluation of its use in 19 cases. $\mathrm{J}$ Am Acad Dermatol 2007;56:960-7.

26. Gourgiotou K, Exadaklyou D, Aroni K, et al. Epidermolysis bullosa acquisita: treatment with intravenous immunoglobulins. J Eur Acad Dermatol Venereol 2002;16:77-80.

27. Crichlow SM, Mortimer NJ, Harman KE. A successful therapeutic trial of rituximab in the treatment of a patient with recalcitrant high-titre epidermolysis bullosa acquisita.
Br J Dermatol 2007;156:194-6.

28. Saha M, Cutler T, Bhogal B, et al. Refractory epidermolysis bullosa acquisita: successful treatment with rituximab. Clin Exp Dermatol 2009;34:e979-e980.

29. Peterson JD, Chan LS. Effectiveness and side effects of anti-CD20 therapy for autoantibody-mediated blistering skin diseases: A comprehensive survey of 71 consecutive patients from the Initial use to 2007. Ther Clin Risk Manag 2009; 5:1-7.

30. Schmidt E, Bröcker E-B, Goebeler M. Rituximab in treatment-resistant autoimmune blistering skin disorders. Clin Rev Allerg Immunol 2008;34:56-64.

31. Chen M, 0'Toole EA, Sanghavi J, et al. The epidermolysis bullosa acquisita antigen (Type VII Collagen) is present in human colon and patients with Chron's disease have Autoantibodies to Type VII Collagen. J Invest Dermatol 2002;6:1059-64.

32. Kabashima R, Hino R, Bito T, et al. Epidermolysis bullosa acquisita associated with psoriasis. Acta Dermatol Venereol 2010; 90:314-6.

33. Jappe U, Zillikens D, Bonnekoh B, Gollnick H. Epidermolysis bullosa acquisita with ultraviolet radiation sensitivity. $\mathrm{Br} \mathrm{J}$ Dermatol 2000;142:517-20. 